\title{
Idosos institucionalizados: prevalência de demências, características demográficas, clínicas e motivos da institucionalização
}

\author{
Ezequiel Vitório Lini", Marlene Doring", Vicente Lucas Moraes Machado"**;, Marilene Rodrigues Portella***
}

\section{Resumo}

Com o objetivo de determinar a prevalência de demências entre idosos institucionalizados e investigar os principais motivos alegados para residirem nas instituições, realizou-se um estudo descritivo com 250 idosos institucionalizados no município de Passo Fundo, Rio Grande do Sul, no período entre julho de 2011 e junho de 2012. Os dados foram coletados por meio de entrevistas com os idosos, com profissionais responsáveis pelas instituições e por análise dos prontuários. Consideraram-se as variáveis demográficas e as relacionadas às condições de saúde. Realizou-se análise descritiva dos dados, utilizando-se medidas de proporção, de tendência central e dispersão. A demência afetava 32,0\% dos idosos, sendo a doença de Alzheimer o diagnóstico mais frequente $(27,5 \%)$. A institucionalização refletiu-se em mais pessoas do sexo feminino, com 63,2\%, outros dados foram: não tinham nenhum ano de estudo, $47,2 \%$ dos idosos, $43,2 \%$ eram solteiros, e a ocupação mais relatada foi do lar $(29,2 \%)$. Em 49,2\% dos idosos institucionalizados, identificaram-se os maiores níveis de dependência (estágios II e III). Os que usavam medicamentos tota- lizavam 96,4\%; os que tinham problemas cardiovasculares, $37,6 \%$, e $74,4 \%$ estavam na instituição porque precisavam de cuidados. A alta taxa de prevalência de demências e o grau de dependência dos idosos institucionalizados que exigem cuidados constantes apontam para a necessidade de suporte às famílias que convivem com a demência, ações preventivas que retardam ao máximo a instalação de dependências e instituições de longa permanência estruturadas e com profissionais especializados para acolher esses idosos.

Palavras-chave: Idoso. Instituição de longa permanência para idosos. Demência.

\section{Introdução}

O crescente número de idosos é uma realidade em países em desenvolvimento, como o Brasil. À medida que esse contingente aumenta, há um acréscimo também na incidência de agravos crônicos (VERAS, 2009). Dentre o rol

* Mestre em Envelhecimento Humano pela Universidade de Passo Fundo. E-mail: ezequielfisio@hotmail.com

*** Docente do Programa de Pós-Graduação em Envelhecimento Humano, Universidade de Passo Fundo. E-mail: doring@upf.br

**** Graduado em Enfermagem pela Universidade de Passo Fundo. E-mail: 109958@upf.br

**** Docente e coordenadora do Programa de Pós-Graduação em Envelhecimento Humano, Universidade de Passo Fundo. E-mail: portella@upf.br

$\rightarrow$ http://dx.doi.org/10.5335/rbceh.2014.4482

Recebido em: 11/11/2014. Aceito em: 11/03/2015 
de doenças crônicas mais frequentes, podemos citar as cardiovasculares, o diabetes e a doença pulmonar obstrutiva crônica. No entanto, algumas doenças têm se agregado a esse grupo devido ao aumento de sobrevida, tais como alguns distúrbios psiconeurológicos, sobretudo as demências (VERAS, 2012).

Estima-se que as demências afetam de $2,0 \%$ a $8,0 \%$ da população acima de 60 anos (WHO, 2012). Na América Latina, a prevalência média das síndromes demenciais é da ordem de $7,1 \%$, com a doença de Alzheimer considerada a causa mais comum (NITRINI et al., 2009).

A demência, termo originado do latim demens (de-privação, mens-inteligência) (MANFRIN; SCHMIDT, 2011), é uma síndrome, geralmente de natureza crônica e progressiva, caracterizada por uma degradação da função cognitiva, ou seja, da capacidade de processar o pensamento, com intensidade acima daquela considerada como normal do envelhecimento (WHO, 2012).

$\mathrm{O}$ início dos sintomas baseia-se na presença do declínio cognitivo persistente do idoso, geralmente progressivo e crônico, que começa a interferir na capacidade do indivíduo de desempenhar suas atividades profissionais ou sociais (NITRINI, 2009). Honig (2011) refere que a concentração dos casos acentua-se nas demências de origem neurodegenerativa, e no fato de que a doença de Alzheimer representa de 60,0\% a 85,0\% dos casos.

Os idosos sofrem ainda com déficits na capacidade de realização das atividades de vida diária (AVDs), necessitando de cuidado diferenciado por seus fami- liares. Alimentar-se, tomar banho ou ir ao banheiro são atos essenciais na vida do ser humano e podem tornar-se árduas tarefas para o portador de alguma demência, principalmente em estágio avançado. Este estudo investiga qual a prevalência de demências nas instituições de longa permanência para idosos (ILPIs) e quais os principais motivos da institucionalização relatados pelos idosos ou por seus cuidadores.

Conhecer a prevalência das principais demências dos idosos poderá contribuir para que a rede de atenção básica de saúde enquadre-se nas novas necessidades e e possam ser criadas estratégias que beneficiem os idosos, que contemplem as necessidades específicas de cada um.

Dessa forma, objetivou-se determinar a prevalência de demências entre idosos residentes nas ILPIs e investigar os principais motivos da institucionalização desses idosos.

\section{Método}

Trata-se de um estudo descritivo com idosos institucionalizados, com base em um recorte da pesquisa: Cenários de cuidados de longa duração: possibilidades avaliativas, interventivas e educacionais na atenção gerontológica.

Das dezenove ILPIs existentes no município, catorze aceitaram participar do estudo. Dessas, apenas três não possuem fins lucrativos, as demais instituições são privadas. A população do estudo foi composta por todos os idosos (250) residentes nas catorze ILPIs do município de Passo Fundo, de ambos os sexos, com idade igual ou superior a 60 anos. 
$\mathrm{Na}$ coleta dos dados, foram realizadas entrevistas com os idosos, com o profissional responsável pela instituição, além de efetivar uma busca ativa nos prontuários dos idosos institucionalizados. Aplicou-se um instrumento estruturado elaborado pelos pesquisadores contendo as variáveis relacionadas aos idosos: idade, sexo, escolaridade, ocupação, estado civil, principal razão de estar morando na instituição e as questões relacionadas às condições de saúde, como: ter demência, o tipo de demência, uso de medicação e grau de dependência. Para investigar as demências, complementaram-se as informações com registros dos prontuários dos residentes institucionalizados.

Para medir o grau de dependência, utilizou-se a definição da Resolução da Diretoria Colegiada da Agência Nacional de Vigilância Sanitária (RDC 283, de 26 de setembro de 2005). Essa resolução considera que o idoso é dependente quando sua condição requer o auxílio de pessoas ou de equipamentos especiais para realização de atividades da vida diária (AVDs). O grau de dependência do idoso foi determinado em três estágios:

a) Grau de Dependência I - idosos independentes, mesmo que requeiram uso de equipamentos de autoajuda;

b) Grau de Dependência II - idosos com dependência em até três atividades de autocuidado para a vida diária tais como: alimentação, mobilidade, higiene; sem comprometimento cognitivo ou com alteração cognitiva controlada;

c) Grau de Dependência III - idosos com dependência que requeiram assistência em todas as atividades de autocuidado para a vida diária e ou com comprometimento cognitivo (ANVISA, 2005).
Após, procedeu-se à análise descritiva dos dados, utilizando-se medidas de proporção, de tendência central e dispersão. Os aspectos éticos foram contemplados com a aprovação do Comitê de Ética em Pesquisa da Universidade de Passo Fundo, Protocolo n. 393/2011. Todos os participantes do estudo assinaram o termo de consentimento livre e esclarecido.

\section{Resultados}

Participaram do estudo 250 idosos institucionalizados, a maioria do sexo feminino $(63,2 \%)$ e com mais de 80 anos $(48,0 \%)$. Uma grande parcela desses idosos $(47,2 \%)$ não tem nenhum estudo, enquanto $30,0 \%$ têm o ensino fundamental. Em relação à paternidade, $43,6 \%$ não tiveram filhos, $39,2 \%$ tiveram dois filhos ou mais. Há $43,2 \%$ de solteiros e $40,0 \%$ de viúvos (Tabela 1). As ocupações anteriores à institucionalização relatadas com maior frequência foram do lar $(29,2 \%)$ e agricultor $(12,8 \%)$. 
Tabela 1: Características demográficas dos idosos institucionalizados, Passo Fundo, 2012

\begin{tabular}{|c|c|c|}
\hline Características & № & $\%$ \\
\hline \multicolumn{3}{|l|}{ Sexo } \\
\hline Masculino & 92 & 36,8 \\
\hline Feminino & 158 & 63,2 \\
\hline \multicolumn{3}{|l|}{ Faixa etária } \\
\hline $60-69$ & 49 & 19,6 \\
\hline $70-79$ & 81 & 32,4 \\
\hline 80 e mais & 120 & 48,0 \\
\hline \multicolumn{3}{|l|}{ Anos de estudo } \\
\hline Nenhum & 118 & 47,2 \\
\hline Fundamental & 75 & 30,0 \\
\hline Ensino médio & 22 & 8,8 \\
\hline Ensino superior & 11 & 4,4 \\
\hline Ignorado & 24 & 9,6 \\
\hline \multicolumn{3}{|c|}{ Aposentado ou pensionista } \\
\hline Sim & 239 & 95,6 \\
\hline Não & 1 & 0,4 \\
\hline Ignorado & 10 & 4,0 \\
\hline \multicolumn{3}{|l|}{ Número de filhos } \\
\hline Nenhum & 109 & 43,6 \\
\hline Um & 35 & 14,0 \\
\hline Dois ou mais & 98 & 39,2 \\
\hline Ignorado & 8 & 3,2 \\
\hline \multicolumn{3}{|l|}{ Estado civil } \\
\hline Solteiro & 108 & 43,2 \\
\hline Casado & 21 & 8,4 \\
\hline Divorciado & 11 & 4,4 \\
\hline Viúvo & 100 & 40,0 \\
\hline Ignorado & 10 & 4,0 \\
\hline
\end{tabular}

Fonte: dados da pesquisa.

Segundo a escala RDC 283, dos idosos, 50,8\% foram classificados com grau de dependência I (ou seja, são considerados independentes, mesmo que necessitem de algum equipamento de ajuda, tais como bengala, andador, óculos, cadeira de rodas, dentre outros com função semelhante), 30,8\% demandaram dependência de grau II, e 18,4\% com grau de dependência III. Expressivo número desses pacientes usava algum tipo de medicamento, totalizando $96,4 \%$ (Tabela 2).

No que se refere às condições clínicas, $37,6 \%$ apresentavam problemas cardiovasculares; $26,4 \%$, osteoporose; $26,0 \%$ apresentaram artrose; $16,8 \%$, sequelas de acidente vascular encefálico (AVE), e 14,0\% declararam ter diabetes (Tabela 2).

Tabela 2: Características clínicas dos idosos institucionalizados, Passo Fundo, 2012

\begin{tabular}{l|r|r}
\hline \multicolumn{1}{|c|}{ Características clínicas } & \multicolumn{1}{|c|}{ № } & $\%$ \\
\hline Demências & & \\
Sim & 80 & 32,0 \\
Não & 170 & 68,0 \\
Grau de dependência & 127 & 50,8 \\
Grau I & 77 & 30,8 \\
Grau II & 46 & 18,4 \\
Grau III & & \\
Uso de medicamentos & 241 & 96,4 \\
Sim & 7 & 2,8 \\
Não & 2 & 0,8 \\
Ignorado & & \\
Problemas cardiovasculares & 94 & 37,6 \\
Sim & 156 & 62,4 \\
Não & & \\
Osteoporose & 66 & 26,4 \\
Sim & 184 & 73,6 \\
Não & & \\
Artrose & 65 & 26,0 \\
Sim & 185 & 74,0 \\
Não & & \\
Sequela de AVE & 42 & 16,8 \\
Sim & 208 & 83,2 \\
Não & & \\
Diabetes & 35 & 14,0 \\
Sim & 215 & 86,0 \\
Não & & \\
\hline
\end{tabular}

Fonte: dados da pesquisa. 
A prevalência de idosos com algum tipo de demência foi $32,0 \%$. Entre as demências diagnosticadas, a doença de Alzheimer apresentou a maior frequência, com $27,5 \%$ (Tabela 3 ).

Tabela 3: Tipos de demências dos idosos institucionalizados, Passo Fundo, 2012

\begin{tabular}{l|r|r}
\hline \multicolumn{1}{c|}{ Demências } & \multicolumn{1}{|c|}{ № } & \multicolumn{1}{c}{$\%$} \\
\hline Alzheimer & 22 & 27,5 \\
Senil & 9 & 11,2 \\
Vascular & 5 & 6,3 \\
Decorrente de Parkinson & 6 & 7,5 \\
Não especificadas & 38 & 47,5 \\
Total & 80 & 100 \\
\hline
\end{tabular}

Fonte: dados da pesquisa.

Entre os motivos que levaram os idosos para a ILPI, observou-se que $74,4 \%$ necessitavam de cuidado, $13,6 \%$ precisavam de companhia, porque viviam só, $4,8 \%$ tinham dificuldades de conviver com os filhos, $4,4 \%$ tinham aporte econômico e social insuficiente, e 2,8\% tinham sofrido maus-tratos.

\section{Discussão}

A prevalência de demência encontrada neste estudo $(32,0 \%)$ está entre quatro e dezesseis vezes acima do previsto pela Organização Mundial da Saúde, que estima taxas entre 2,0 a $8,0 \%$ de demências na população idosa mundial (WHO, 2012). No entanto, a Organização Mundial da Saúde identifica os idosos no todo, ou seja, prevê uma estimativa em toda a população idosa, residentes em seus domicílios, na comunidade e não somente nas ILPIs, como tratado nesta pesquisa.
Os diagnósticos encontrados estavam documentados em prontuários, confirmados, assim, pelas equipes de saúde de cada instituição. Chehuen Neto et al. (2012), ao traçarem o perfil epidemiológico de 366 idosos residentes em oito ILPIs de Juiz de Fora - MG, identificaram que $26,5 \%$ dos idosos apresentaram diagnóstico de demência.

Muitas investigações utilizam instrumentos de rastreio para demências, a exemplo de Álvares, Lima e Silva (2010), que, ao aplicarem o miniexame do estado mental (MEEM) em dezenove ILPIs, identificaram desempenho abaixo do esperado em $55,6 \%$ dos idosos. Entretanto, é necessário enfatizar que o MEEM permite a avaliação da função cognitiva e o rastreamento de quadros demenciais, mas sozinho não pode ser utilizado para diagnosticar demência, e a confirmação diagnóstica exige uma avaliação abrangente por uma equipe de saúde (BRASIL, 2006).

Entre as demências identificadas neste estudo, a mais frequente foi a doença de Alzheimer. Nessa busca por diagnósticos, deparou-se com a dificuldade de classificação das demências, pois em $47,5 \%$ dos casos, a classificação era não especificada. Essa dificuldade em identificar a demência é referida por Fornari et al. (2010). Os autores estabelecem, em sua revisão, que o diagnóstico das síndromes demenciais é complexo, com fundamentação em largo arcabouço, tais como a prática clínica, anamnese e exame físico, testes cognitivos e neuropsicológicos padronizados, neuroimagem e exames laboratoriais. Por fim, concluem que o diagnóstico definitivo para demên- 
cias degenerativas ainda dependeria de análises histopatológicas de materiais de autópsia.

A alta prevalência de demências encontrada nesta pesquisa indica que as demências podem ser consideradas um dos fatores preditivos para institucionalização. Recente estudo de coorte australiano, que objetivou avaliar uma série de fatores de risco de institucionalização, acompanhou homens com 70 anos ou mais, entre 2005 e 2007, com avaliações periódicas e concluiu que os preditores mais fortes de institucionalização foram demência, comprometimento cognitivo, dependência para atividades básicas e instrumentais de vida diária. Os idosos com demência apresentaram risco seis vezes maior de serem institucionalizados do que os sem demência (GNJIDIC et al., 2012).

No que se refere aos motivos que levaram os idosos para as ILPIs, evidenciou-se que em $74,4 \%$ dos casos o motivo principal foi a necessidade de cuidados. Cabe ressaltar que a demência é uma das principais causas de dependência e incapacidade nos idosos em todo o mundo (WHO, 2012).

Outro fator preponderante é que $49,2 \%$ dos idosos analisados apresentaram grau de dependência II ou III para as atividades de vida diária, estágios considerados de maior demanda por auxílio. Essa dependência pode ser oriunda das demências (presente em 32,0\% dos idosos), das sequelas de AVE (16,8\%), da diminuição das habilidades esperadas em idosos com 80 anos ou mais $(48,0 \%)$, dentre outras, que exigem cuidados em maior ou menor grau. $O$ processo de perda da independência começa pelas tarefas mais complexas, com maior exigência, - como banhar-se, - e progride até a dependência para alimentar-se (MORAES, 2012). A dependência para as AVDs foi considerada fator de risco à institucionalização para Del Duca et al. (2012), que encontraram dependência para, no mínimo, uma atividade da vida diária em $79,4 \%$ dos idosos institucionalizados. Estudo longitudinal realizado em Hong Kong acompanhou, durante doze meses, 749 indivíduos com idade $\geq 65$ anos. Todos apresentavam déficit de funcionalidade, com ou sem declínio cognitivo. No período estudado, 80 idosos foram institucionalizados e 0 comprometimento cognitivo mostrou-se indicativo de institucionalização. Quando o comprometimento funcional esteve associado, a taxa de institucionalização praticamente triplicou. Os autores identificaram ainda, que a presença de cuidador domiciliar reduziu em $70,0 \%$ as chances de institucionalização neste período (CHAU et al., 2012).

$\mathrm{O}$ agravamento de algumas doenças motiva a institucionalização, em especial a doença de Alzheimer. Entrevistas com cuidadores de idosos com Parkinson e Alzheimer, realizado na Universidade de Indiana, Estados Unidos, indicou que os idosos com Alzheimer são mais propensos à institucionalização, porque o cuidado em domicílio é mais complexo e desgastante devido às dependências e à necessidade de atenção contínua (MCLENNON; HABERMANN; DAVIS, 2010).

Diante dessa necessidade de cuidados, vale destacar os seguintes dados: $43,2 \%$ dos idosos eram solteiros, $40,0 \%$ viúvos e 43,6\% não tinham filhos. Esses 
resultados indicam a dificuldade de um cuidador familiar para esse idoso. Para Küchemann (2012), o cuidado aos idosos tradicionalmente é ofertado pela família, no entanto, o autor destaca as mudanças no perfil familiar, com famílias cada vez menores e com separações, mudanças nem sempre favoráveis aos idosos. Para Silva, Passos e Barreto (2012), os cuidadores são em sua maioria mulheres (filhas) e mais de a metade trabalha fora de casa, o que caracteriza sobrecarga de funções, com redução das horas de sono, ansiedade e depressão. Assim, quando existe cuidador familiar, comumente está sobrecarregado. $\mathrm{Na}$ inexistência de um cuidador familiar, a alternativa pode ser a contratação de profissionais especializados, considerada financeiramente inviável para a maioria das famílias em vista da condição socioeconômica (GURGEL; OLIVEIRA; SALLES, 2012).

As limitações deste estudo, como a imprecisão diagnóstica nos quadros demenciais, dificulta a caracterização das principais demências, o que não impossibilita, no entanto, alcançar o objetivo de determinar a prevalência dessas demências.

\section{Conclusões}

A alta taxa de prevalência de demência, grande parte sem diagnóstico preciso quanto à classificação do quadro demencial, aponta para a necessidade de instituições de longa permanência para idosos com estruturas adequadas e profissionais especializados para acolher, acompanhar e proporcionar diagnósticos precisos.
$\mathrm{O}$ fato de que praticamente metade dos idosos avaliados possui dependência - necessitando de cuidados especializados, muitas vezes de forma integral - destaca-se como fator desencadeante de institucionalização. Esses resultados potencializam a necessidade de se rever estratégias de cuidados, seja nos domicílios, na comunidade, seja nas instituições de longa permanência.

Para as famílias que convivem com a demência, deve-se disponibilizar suporte diferenciado e ações preventivas que retardem ao máximo a instalação de dependências, para que o idoso permaneça em seu domicílio, junto de seus familiares, o maior tempo possível.

Ainda, as instituições de longa permanência para idosos devem estar preparadas, de forma sustentável e com um atendimento qualificado, requisito esse que, em grande parte, pode ser atribuído aos serviços dispensados pela equipe de saúde multiprofissional.

\section{Institutionalized aged people: prevalence of dementia, demographic characteristics and reasons of institutionalization}

\section{Abstract}

Aiming to identify the prevalence of dementia in long-term care institutions for elderly people and verify the reasons for institutionalization, we performed a cross-sectional study of 250 institutionalized elderly in Passo Fundo, Rio Grande do Sul, during the period from July 2011 until June 2012. The data were collected through interviews with the professional responsible for the institution and by ac- 
tive search in the records of the inpatients. Were considered demographic variables and related health conditions. Dementia was present in $32.0 \%$ of the elderly, being Alzheimer's disease the most obvious diagnosis $(27.5 \%)$. The institutionalization was more common in females, with $63.2 \%$, the elderly that did not have any year of study were $47.2 \%$, among the cases, $43.2 \%$ were single and the most reported occupation was housewife (29.2\%). In $49.2 \%$ of the inpatients were identified the highest levels of dependence (stages II and III). Those who used medicines totaled $96.4 \%$. $37.6 \%$ had cardiovascular problems and $74.4 \%$ were in the institution because they needed care. The high prevalence of dementia and the degree of dependence of the institutionalized elderly people who demand constant care point to the need of structured long-term care facilities with specialized professionals to shelter the patients.

Keywords: Elderly people. Long-term care institutions for the elderly. Dementia.

\section{Agradecimento}

À Fundação de Amparo à Pesquisa do Estado do Rio Grande do Sul (Fapergs), pela bolsa concedida.

\section{Referências}

ÁlVARES, L. M.; LIMA, R. C.; SILVA, R. A. Ocorrência de quedas em idosos residentes em instituições de longa permanência em Pelotas, Rio Grande do Sul, Brasil. Cadernos de Saúde Pública, Rio de Janeiro, v. 26, n. 1, p. 31-40, jan. 2010.

ANVISA - AGÊNCIA NACIONAL DE VIGILÂNCIA SANITÁRIA. Resolução da Diretoria Colegiada - RDC/ANVISA n. 283, de 26 de setembro de 2005. Disponível em: <http:// www.anvisa.gov.br>. Acesso em: 20 fev. 2014.
BRASIL. Envelhecimento e saúde da pessoa idosa. Brasília, DF: Ministério da Saúde, 2006. Cadernos de Atenção Básica, n. 19. Disponível em: <http://189.28.128.100/dab/ docs/publicacoes/cadernos_ab/abcad19.pdf > . Acesso em: 8 fev. 2014.

CHAU, P. H. et al. Usage of Community Services and Domestic Helpers Predicted Institutionalization of Elders Having Functional or cognitive impairments: a 12-month longitudinal study in Hong Kong. Journal of the American Medical Directors Association, Washington, v. 13, n. 2, p. 169-175, Feb. 2012.

CHEHUEN NETO, J. A. et al. Perfil epidemiológico dos idosos institucionalizados em Juiz de Fora. HU Revista, Juiz de Fora, v. 37, n. 2, p. 207-216, abr./jun. 2011.

DEL DUCA, G. F. et al. Indicadores da institucionalização de idosos: estudo de casos e controles. Revista Saúde Pública, São Paulo, v. 46 , n. 1, p. 147-153, fev. 2012.

FORNARI, L. H. T. et al. As diversas faces da síndrome demencial: como diagnosticar clinicamente? Scientia Médica, Porto Alegre, v. 20, n. 2, p. 185-193, abr./jun. 2010.

GNJIDIC, D. et al. Mild Cognitive Impairment Predicts Institutionalization among Older Men: A Population-Based Cohort Study. PLoS One, San Francisco, USA, v. 7, n. 9, p. 1-8, Sept. 2012.

GURGEL, D. A.; OLIVEIRA, F. P. A.; SALLES, H. S. A. Cuidador de idoso doente crônico e suas dificuldades. Revista Kairós Gerontologia, São Paulo, v. 15, n. 2, p. 129143, mar. 2012.

HONIG, L. S. Demência considerações gerais. In: ROWLAND, L. P.; PEDLEY, T. A. Tratado de Neurologia. 12. ed. São Paulo: Guanabara Koogan, 2011. p. 717-719.

KÜCHEMANN, B. A. Envelhecimento populacional, cuidado e cidadania: velhos dilemas e novos desafios. Sociedade e Estado, Brasília, DF, v. 27, n. 1, p. 165-180, jan./abr. 2012. 
MANFRIN, A.; SCHMIDT, S. L. Diagnóstico diferencial das demências. In: FREITAS, E. V. et al. Tratado de Geriatria e Gerontologia. 3. ed. Rio de Janeiro: Guanabara Koogan, 2011. p. 157-168.

MCLENNON, S. M.; HABERMANN, B.; DAVIS, L. L. Deciding to institutionalize: why do family members cease caregiving at home? The Journal of Neuroscience Nursing. Journal of the American Association of Neuroscience Nurses, Glenview, USA, v. 42, n. 2, p. 95-103, Apr. 2010.

MORAES, E. N. de. Atenção à saúde do idoso: aspectos conceituais. Brasília, DF: Ministério da Saúde, 2012. Disponível em: <http://apsredes.org/site2012/wp-content/ uploads/2012/05/Saude-do-Idoso-WEB1. pdf>. Acesso em: 11 jul. 2013.

NITRINI, R. Distúrbios cognitivos. In: MARTINS, M. A. et al. Clínica Médica: doenças dos olhos, doenças dos ouvidos, nariz e garganta, neurologia, transtornos mentais. São Paulo: Manole, 2009. p. 242-255.

NITRINI, R. et al. Prevalence of dementia in Latin America: a collaborative study of population-based cohorts. International Psychogeriatrics, New York, v. 21, n. 4, p. 622-630, Aug. 2009.

SILVA, C. F. da; PASSOS, V. M. de A.; BARRETO, S. M. Frequência e repercussão da sobrecarga de cuidadoras familiares de idosos com demência. Revista Brasileira de Geriatria e Gerontologia, Rio de Janeiro, v. 15, n. 4, p. 707-731, out./dez. 2012.

VERAS, R. P. Envelhecimento populacional contemporâneo: demandas, desafios e inovações. Revista de Saúde Pública, São Paulo, v. 43, n. 3, p. 548-554, maio/jun. 2009.

VERAS, R. P. Um modelo em que todos ganham: mudar e inovar, desafios para o enfrentamento das doenças crônicas entre os idosos, Acta Scientiarum. Human and Social Sciences, Maringá, v. 34, n. 1, p. 3-8, jan./jun. 2012.
WHO - WORLD HEALTH ORGANIZATION. Dementia. WHO Fact Sheets, 2012. Disponível em: <www.who.int/mediacentre/ factsheets/fs362/en/>. Acesso em: 22 mar. 2014. 